\title{
Periodic Solutions for a System of Nonlinear Neutral Functional Difference Equations with Two Functional Delays
}

\author{
Mouataz Billah Mesmouli, Abdelouaheb Ardjouni, \\ AND AHCEne DJOUdi
}

\begin{abstract}
In this paper, we study the existence and uniqueness of periodic solutions of the system of nonlinear neutral difference equations

$$
+G(n, x(n), x(n-g(n))) \text {. }
$$$$
\Delta x(n)=A(n) x(n-\tau(n))+\Delta Q(n, x(n-g(n)))
$$

By using Krasnoselski's fixed point theorem we obtain the existence of periodic solution and by contraction mapping principle we obtain the uniqueness. An example is given to illustrate our result. Our results extend and generalize the work [13].
\end{abstract}

\section{INTRODUCTION}

A qualitative analysis such as periodicity and stability of solutions of neutral difference equations which the delay has been studied extensively by many authors, we refer the readers to [1]-[5], [7]-[9], [10, 12, 13] and references therein for a wealth of reference materials on the subject.

In 2005, Y. N. Raffoul in [13] studied the existence and uniqueness of periodic solutions for the system of nonlinear neutral functional difference equations

$$
\begin{gathered}
\Delta x(n)=A(n) x(n)+\Delta Q(n, x(n-g(n))) \\
+G(n, x(n), x(n-g(n))) .
\end{gathered}
$$

By employing the Krasnoselskii's fixed point theorem, the author obtained existence results for periodic solutions. Also, the author used the contraction mapping principle to show the uniqueness of periodic solutions of (1).

2010 Mathematics Subject Classification. 35A08, 34A34, 34K40, 35B10, 39A70.

Key words and phrases. Krasnoselskii's theorem, Contraction, Neutral difference equation, Periodic solution, Fundamental matrix solution. 
In the current paper, we study the existence and uniqueness of periodic solutions of the system of nonlinear neutral difference equations

$$
\begin{gathered}
\Delta x(n)=A(n) x(n-\tau(n))+\Delta Q(n, x(n-g(n))) \\
+G(n, x(n), x(n-g(n))),
\end{gathered}
$$

where $A(\cdot)$ is $N \times N$ matrix with sequences as its elements, $\tau, g: \mathbb{Z} \rightarrow \mathbb{Z}^{+}$ are scalar and the functions $Q: \mathbb{Z} \times \mathbb{R}^{N} \rightarrow \mathbb{R}^{N}$ and $G: \mathbb{Z} \times \mathbb{R}^{N} \times \mathbb{R}^{N} \rightarrow$ $\mathbb{R}^{N}$ are continuous in $x$. The sets $\mathbb{Z}$ and $\mathbb{Z}^{+}$denote the integers and the nonnegative integers, respectively. In the analysis we use the fundamental matrix solution of $\Delta x(n)=A(n) x(n)$ to invert the system (2). Then we employ the Krasnoselskii's fixed point theorem to show the existence of periodic solutions of system (2). The obtained mapping is the sum of two mappings, one is a compact operator and the other is a contraction. Also, transforming system (2) to a fixed point problem enables us to show the uniqueness of the periodic solution by appealing to the contraction mapping principle.

The organization of this paper is as follows. In Section 2, we present the inversion of (2) and the fixed point theorems that we employ to help us show the existence and uniqueness of periodic solutions to system (2). In Section 3 , we present our main results with an example.

\section{Preliminaries}

For the definitions of the different notions used throughout this paper we refer, for example $[6,7,10,11,14]$.

For $T>1$ define

$$
\mathcal{C}_{T}=\left\{\phi: \phi \in C\left(\mathbb{Z}, \mathbb{R}^{N}\right), \phi(n+T)=\phi(n)\right\},
$$

where $C\left(\mathbb{Z}, \mathbb{R}^{N}\right)$ is the space of all $N$-vector continuous functions. Then $\mathcal{C}_{T}$ is a Banach space when it is endowed with the supremum norm

$$
\|x(\cdot)\|=\max _{n \in \mathbb{Z}}\left|x(n)=\max _{n \in[0, T-1] \cap \mathbb{Z}}\right| x(n) \mid,
$$

Note that $\mathcal{C}_{T}$ is equivalent to the Euclidean space $\mathbb{R}^{N T}$, where $|\cdot|$ denotes the infinity norm for $x \in \mathbb{R}^{N}$. Also, if $A$ is an $N \times N$ real matrix, then we define the norm of $A$ by

$$
|A|=\max _{1 \leq i \leq N} \sum_{j=1}^{N}\left|a_{i j}\right| .
$$

Definition 2.1. If the matrix $B(\cdot)$ is periodic of period $T$, then the linear system

$$
y(n+1)=B(n) y(n),
$$

is said to be noncritical with respect to $T$, if it has no periodic solution of period $T$ except the trivial solution $y=0$. 
In this paper we assume that

$$
\begin{aligned}
A(n+T) & =A(n), \\
\tau(n+T) & =\tau(n) \geq \tau^{*}>0, \\
g(n+T) & =g(n) \geq g^{*}>0,
\end{aligned}
$$

where $\tau^{*}, g^{*}$ are constant. For $n \in \mathbb{Z}, x, y, z, w \in \mathbb{R}^{N}$, the functions $Q(n, x)$ and $G(n, x, y)$ are periodic in $n$ of period $T$, they are also globally Lipschitz continuous in $x$ and in $x$ and $y$, respectively. That is

$$
Q(n+T, x)=Q(n, x), G(n+T, x, y)=G(n, x, y),
$$

and there are positive constants $k_{1}, k_{2}, k_{3}$ such that

$$
\begin{gathered}
|Q(n, x)-Q(n, y)| \leq k_{1}\|x-y\|, \\
|G(n, x, y)-G(n, z, w)| \leq k_{2}\|x-z\|+k_{3}\|y-w\| .
\end{gathered}
$$

Throughout this paper it is assumed that the matrix $B(n)=I+A(n)$ is nonsingular and the system (3) is noncritical, where $I$ is the

$N \times N$ identity matrix. Also, if $x(\cdot)$ is a sequence, then the forward operator $E$ is defined as $\operatorname{Ex}(n)=x(n+1)$. Now, we state some known results about system (3). Let $K(n)$ represent the fundamental matrix of (3) with $K(0)=I$, then:

a. $\operatorname{det} K(n) \neq 0$.

b. $K(n+T)=B(n) K(n)$ and $K^{-1}(n+T)=K^{-1}(n) B^{-1}(n)$.

c. System (3) is noncritical if and only if $\operatorname{det}(I-K(T)) \neq 0$.

d. There exists a nonsingular matrix $L$ such that

$$
\begin{aligned}
& K(n+T)=B(n) K(n) L^{T} \text { and } \\
& K^{-1}(n+T)=L^{-T} K^{-1}(n) .
\end{aligned}
$$

The following lemma is fundamental to our results.

Lemma 2.1. Suppose (4) and (5) hold. If $x \in \mathcal{C}_{T}$, then $x$ is a solution of the equation (2) if and only if

$$
\begin{aligned}
& x(n)=Q(n, x(n-g(n)))-\sum_{s=n-\tau(n)}^{n-1} A(s) x(s) \\
& +\sum_{s=n}^{n+T-1} \mathcal{G}(n, s)\left[A(s)\left(Q(s, x(s-g(s)))-\sum_{u=s-\tau(s)}^{s-1} A(u) x(u)\right)\right. \\
& +F(s) x(s-\tau(s))+G(s, x(s), x(s-g(s)))],
\end{aligned}
$$

where

$$
\mathcal{G}(n, s)=K(n)\left(K^{-1}(T)-I\right)^{-1} K^{-1}(s)\left(I-A(s) B^{-1}(s)\right),
$$


and

$$
F(n)=A(n)-(1-\Delta \tau(n)) A(n-\tau(n)) .
$$

Proof. Let $x \in \mathcal{C}_{T}$ be a solution of (2) and $K(\cdot)$ is a fundamental matrix of solutions for (3). Rewrite the equation (2) as

$$
\begin{aligned}
\Delta x(n)=A & (n) x(n)-A(n) x(n)+A(n) x(n-\tau(n)) \\
& +\Delta Q(n, x(n-g(n)))+G(n, x(n), x(n-g(n))) \\
=A & (n) x(n)-\Delta_{n} \sum_{u=n-\tau(n)}^{n-1} A(u) x(u) \\
& +[A(n)-(1-\Delta \tau(n)) A(n-\tau(n))] x(n-\tau(n)) \\
& +\Delta Q(n, x(n-g(n)))+G(n, x(n), x(n-g(n))) .
\end{aligned}
$$

We put $A(n)-(1-\Delta \tau(n)) A(n-\tau(n))=F(n)$, we obtain

$$
\begin{gathered}
\Delta\left[x(n)-Q(n, x(n-g(n)))+\sum_{u=n-\tau(n)}^{n-1} A(u) x(u)\right] \\
=A(n)\left[x(n)-Q(n, x(n-g(n)))+\sum_{u=n-\tau(n)}^{n-1} A(u) x(u)\right] \\
\quad+A(n)\left[Q(n, x(n-g(n)))-\sum_{u=n-\tau(n)}^{n-1} A(u) x(u)\right] \\
\quad+F(n) x(n-\tau(n))+G(n, x(n), x(n-g(n))) .
\end{gathered}
$$

Since $K(n) K^{-1}(n)=I$, it follows that

$$
\begin{aligned}
0 & =\Delta\left[K(n) K^{-1}(n)\right] \\
& =A(n) K(n) K^{-1}(n) B^{-1}(n)+K(n) \Delta K^{-1}(n) \\
& =A(n) B^{-1}(n)+K(n) \Delta K^{-1}(n) .
\end{aligned}
$$

This implies

$$
\Delta K^{-1}(n)=-K^{-1}(n) A(n) B^{-1}(n)
$$


If $x(\cdot)$ is a solution of $(2)$ with $x(0)=x_{0}$, then

$$
\begin{aligned}
& \Delta\left[K^{-1}(n)\left(x(n)-Q(n, x(n-g(n)))+\sum_{u=n-\tau(n)}^{n-1} A(u) x(u)\right)\right] \\
& =\Delta K^{-1}(n) E\left[x(n)-Q(n, x(n-g(n)))+\sum_{u=n-\tau(n)}^{n-1} A(u) x(u)\right] \\
& +K^{-1}(n) \Delta\left[x(n)-Q(n, x(n-g(n)))+\sum_{u=n-\tau(n)}^{n-1} A(u) x(u)\right] .
\end{aligned}
$$

Thus

$$
\begin{aligned}
& \Delta\left[K^{-1}(n)\left(x(n)-Q(n, x(n-g(n)))+\sum_{u=n-\tau(n)}^{n-1} A(u) x(u)\right)\right] \\
& =-K^{-1}(n) A(n) B^{-1}(n) \\
& \times\left[B(n)\left(x(n)-Q(n, x(n-g(n)))+\sum_{u=n-\tau(n)}^{n-1} A(u) x(u)\right)\right. \\
& +A(n)\left(Q(n, x(n-g(n)))-\sum_{u=n-\tau(n)}^{n-1} A(u) x(u)\right) \\
& +F(n) x(n-\tau(n))+G(n, x(n), x(n-g(n)))] \\
& +K^{-1}(n) A(n)\left(x(n)-Q(n, x(n-g(n)))+\sum_{u=n-\tau(n)}^{n-1} A(u) x(u)\right) \\
& +K^{-1}(n)\left[A(n)\left(Q(n, x(n-g(n)))-\sum_{u=n-\tau(n)}^{n-1} A(u) x(u)\right)\right. \\
& +F(n) x(n-\tau(n))+G(n, x(n), x(n-g(n)))] \\
& =K^{-1}(n)\left(I-A(n) B^{-1}(n)\right) \\
& \times\left[A(n)\left(Q(n, x(n-g(n)))-\sum_{u=n-\tau(n)}^{n-1} A(u) x(u)\right)\right. \\
& +F(n) x(n-\tau(n))+G(n, x(n), x(n-g(n)))] \text {. }
\end{aligned}
$$


Summing of the above equation from 0 to $n-1$ yields

$$
\begin{aligned}
x(n)=Q & (n, x(n-g(n)))-\sum_{s=n-\tau(n)}^{n-1} A(s) x(s) \\
& +K(n)\left(x(0)-Q(0, x(0-g(0)))+\sum_{s=-\tau(0)}^{-1} A(s) x(s)\right) \\
& +K(n) \sum_{s=0}^{n-1} K^{-1}(s)\left(I-A(s) B^{-1}(s)\right) \\
\times & {\left[A(s)\left(Q(s, x(s-g(s)))-\sum_{u=s-\tau(s)}^{s-1} A(u) x(u)\right)\right.} \\
& +(F(s) x(s-\tau(s))+G(s, x(s), x(s-g(s))))] .
\end{aligned}
$$

Since $x(T)=x_{0}=x(0)$, using (11) we get

$$
\begin{aligned}
& x(0)-Q(0, x(-g(0)))+\sum_{s=-\tau(0)}^{-1} A(s) x(s) \\
& =(I-K(T))^{-1} \sum_{s=0}^{T-1} K(T) K^{-1}(s)\left(I-A(s) B^{-1}(s)\right) \\
& \times\left[A(s)\left(Q(s, x(s-g(s)))-\sum_{u=s-\tau(s)}^{s-1} A(u) x(u)\right)\right. \\
& \quad+(F(s) x(s-\tau(s))+G(s, x(s), x(s-g(s))))] .
\end{aligned}
$$

A substitution of (12) into (11) yields

$$
\begin{aligned}
x(n)= & Q(n, x(n-g(n)))-\sum_{s=n-\tau(n)}^{n-1} A(s) x(s) \\
& +K(n)(I-K(T))^{-1} \sum_{s=0}^{T-1} K(T) K^{-1}(s)\left(I-A(s) B^{-1}(s)\right) \\
\times & {\left[A(s)\left(Q(s, x(s-g(s)))-\sum_{u=s-\tau(s)}^{s-1} A(u) x(u)\right)+\right.}
\end{aligned}
$$




$$
\begin{aligned}
& +(F(s) x(s-\tau(s))+G(s, x(s), x(s-g(s))))] \\
& +K(n) \sum_{s=0}^{n-1} K^{-1}(s)\left(I-A(s) B^{-1}(s)\right) \\
\times & {\left[A(s)\left(Q(s, x(s-g(s)))-\sum_{u=s-\tau(s)}^{s-1} A(u) x(u)\right)\right.} \\
& +(F(s) x(s-\tau(s))+G(s, x(s), x(s-g(s))))] .
\end{aligned}
$$

Now, we will show that (13) is equivalent to (8). Since

$$
\begin{aligned}
(I-K(T))^{-1} & =\left(K(T)\left(K(T)^{-1}-I\right)\right)^{-1} \\
& =\left(K(T)^{-1}-I\right)^{-1} K(T)^{-1}
\end{aligned}
$$

Then the equations (13) becomes

$$
\begin{aligned}
x(n)= & Q(n, x(n-g(n)))-\sum_{s=n-\tau(n)}^{n-1} A(s) x(s) \\
& +K(n)\left(K(T)^{-1}-I\right)^{-1} \sum_{s=0}^{T-1} K^{-1}(s)\left(I-A(s) B^{-1}(s)\right) \\
\times & {\left[A(s)\left(Q(s, x(s-g(s)))-\sum_{u=s-\tau(s)}^{s-1} A(u) x(u)\right)\right.} \\
& +(F(s) x(s-\tau(s))+G(s, x(s), x(s-g(s))))] \\
& +\sum_{s=0}^{n-1} K(n) K^{-1}(s)\left(I-A(s) B^{-1}(s)\right) \\
\times & {\left[A(s)\left(Q(s, x(s-g(s)))-\sum_{u=s-\tau(s)}^{s-1} A(u) x(u)\right)\right.} \\
& +(F(s) x(s-\tau(s))+G(s, x(s), x(s-g(s))))] .
\end{aligned}
$$

For the sake of simplicity, we let

$$
\begin{aligned}
D(s)=\left(I-A(s) B^{-1}(s)\right)\left[A(s)\left(Q(s, x(s-g(s)))-\sum_{u=s-\tau(s)}^{s-1} A(u) x(u)\right)\right. \\
+(F(s) x(s-\tau(s))+G(s, x(s), x(s-g(s))))],
\end{aligned}
$$


then

$$
\begin{aligned}
x(n)=Q & (n, x(n-g(n)))-\sum_{s=n-\tau(n)}^{n-1} A(s) x(s) \\
& +K(n)\left(K(T)^{-1}-I\right)^{-1} \\
\times & {\left[\sum_{s=0}^{T-1} K^{-1}(s) D(s)+\sum_{s=0}^{n-1}\left(K(T)^{-1}-I\right) K^{-1}(s) D(s)\right] } \\
= & (n, x(n-g(n)))-\sum_{s=n-\tau(n)}^{n-1} A(s) x(s) \\
& +K(n)\left(K(T)^{-1}-I\right)^{-1}\left[\sum_{s=0}^{T-1} K^{-1}(s) D(s)\right. \\
& \left.+\sum_{s=0}^{n-1} K(T)^{-1} K^{-1}(s) D(s)-\sum_{s=0}^{n-1} K^{-1}(s) D(s)\right] .
\end{aligned}
$$

Thus

$$
\begin{aligned}
x(n)= & Q(n, x(n-g(n)))-\sum_{s=n-\tau(n)}^{n-1} A(s) x(s) \\
& +K(n)\left(K(T)^{-1}-I\right)^{-1} \\
\times & {\left[-\sum_{s=T}^{n-1} K^{-1}(s) D(s)+\sum_{s=0}^{n-1} K(T)^{-1} K^{-1}(s) D(s)\right] . }
\end{aligned}
$$

By letting $s=v-T$ and $U(T)=\left(K(T)^{-1}-I\right)^{-1}$, the above expression yields

$$
\begin{aligned}
x(n)=Q & (n, x(n-g(n)))-\sum_{s=n-\tau(n)}^{n-1} A(s) x(s) \\
& +K(n)\left(K(T)^{-1}-I\right)^{-1}\left[-\sum_{s=T}^{n-1} K^{-1}(s) D(s)\right. \\
& \left.+\sum_{v=T}^{T+n-1} K(T)^{-1} K^{-1}(v-T) D(v-T)\right] .
\end{aligned}
$$

By (d) we have $K(n-T)=K(n) L^{-T}$ and $K(T)=L^{T}$. Hence,

$$
K^{-1}(T) K^{-1}(v-T)=K^{-1}(v) .
$$


Consequently, since (4) and (5) hold, (14) becomes

$$
\begin{aligned}
x(n)= & Q(n, x(n-g(n)))-\sum_{s=n-\tau(n)}^{n-1} A(s) x(s) \\
& +K(n)\left(K(T)^{-1}-I\right)^{-1} \\
\times & {\left[-\sum_{s=T}^{n-1} K^{-1}(s) D(s)+\sum_{s=T}^{T+n-1} K(T)^{-1} K^{-1}(s) D(s)\right] } \\
= & Q(n, x(n-g(n)))-\sum_{s=n-\tau(n)}^{n-1} A(s) x(s) \\
& +K(n)\left(K(T)^{-1}-I\right)^{-1} \sum_{s=n}^{n+T-1} K(T)^{-1} K^{-1}(s) D(s) .
\end{aligned}
$$

The converse implication is easily obtained and the proof is complete.

We end this section by stating the fixed point theorems that we employ to help us show the existence and uniqueness of periodic solutions to equation (2); see $[6,14]$.

Theorem 2.1 (Contraction Mapping Principle). Let $(\mathcal{X}, \rho)$ a complete metric space and let $P: \mathcal{X} \rightarrow \mathcal{X}$. If there is a constant $\alpha<1$ such that for $x, y \in \mathcal{X}$ we have

$$
\rho(P x, P y) \leq \alpha \rho(x, y),
$$

then there is one and only one point $z \in \mathcal{X}$ with $P z=z$.

Krasnoselskii (see [14]) combined the contraction mapping theorem and Shauder's theorem and formulated the following hybrid result.

Theorem 2.2 (Krasnoselskii). Let $\mathbb{M}$ be a closed bounded convex nonempty subset of a Banach space $(\mathcal{X},\|\cdot\|)$. Suppose that $R$ and $S$ map $\mathbb{M}$ into $\mathcal{X}$ such that

(i) $R$ is compact and continuous,

(ii) $S$ is a contraction mapping,

(iii) $x, y \in \mathbb{M}$, implies $R x+S y \in \mathbb{M}$,

then there exists $z \in \mathbb{M}$ with $z=R z+S z$.

\section{Existence and Uniqueness of Periodic Solution}

By applying Theorems 2.1 and 2.2, we obtain in this Section the existence and the uniqueness of the periodic solution of (2). So, let a Banach space $\left(\mathcal{C}_{T},\|\cdot\|\right)$, a closed bounded convex subset of $\mathcal{C}_{T}$,

$$
\mathcal{M}=\left\{\varphi \in \mathcal{C}_{T},\|\varphi\| \leq L\right\},
$$


with $L>0$, and by the Lemma 2.1, let a mapping $\mathcal{H}$ given by

$$
\begin{aligned}
&(\mathcal{H} \varphi)(n)=Q(n, \varphi(n-g(n)))-\sum_{s=n-\tau(n)}^{n-1} A(s) \varphi(s) \\
&(17) \quad+\sum_{s=n}^{n+T} \mathcal{G}(n, s)\left[A(s)\left(Q(s, \varphi(s-g(s)))-\sum_{u=s-\tau(s)}^{s-1} A(u) \varphi(u)\right)\right. \\
&+F(s) \varphi(s-\tau(s))+G(s, \varphi(s), \varphi(s-g(s)))] .
\end{aligned}
$$

Therefore, we express equation (17) as

$$
\mathcal{H} \varphi=\mathcal{R} \varphi+\mathcal{S} \varphi
$$

where $\mathcal{R}$ and $\mathcal{S}$ are given by

$$
\begin{aligned}
(\mathcal{R} \varphi)(n) & =\sum_{s=n}^{n+T} \mathcal{G}(n, s)\left[A(s)\left(Q(s, \varphi(s-g(s)))-\sum_{u=s-\tau(s)}^{s-1} A(u) \varphi(u)\right)\right. \\
(18) & +F(s) \varphi(s-\tau(s))+G(s, \varphi(s), \varphi(s-g(s)))],
\end{aligned}
$$

and

$$
(\mathcal{S} \varphi)(n)=Q(n, \varphi(n-g(n)))-\sum_{s=n-\tau(n)}^{n-1} A(s) \varphi(s) .
$$

By a series of steps we will prove the fulfillment of $(i),(i i)$ and (iii) in Theorem 2.2. So that, since $\varphi \in \mathcal{C}_{T}$, (4) and (5) hold, we have for $\varphi \in \mathcal{M}$

$$
(\mathcal{R} \varphi)(n+T)=(\mathcal{R} \varphi)(n) \text { and } \mathcal{R} \varphi \in C\left(\mathbb{Z}, \mathbb{R}^{N}\right) \Longrightarrow(\mathcal{R} \mathcal{M}) \subset \mathcal{C}_{T},
$$

and

$$
(\mathcal{S} \varphi)(n+T)=(\mathcal{S} \varphi)(n) \text { and } \mathcal{R} \varphi \in C\left(\mathbb{Z}, \mathbb{R}^{N}\right) \Longrightarrow(\mathcal{S M}) \subset \mathcal{C}_{T}
$$

Lemma 3.1. Suppose (4)-(7) hold. If $\mathcal{R}$ is defined by (18), then $\mathcal{R}$ is continuous and the image of $\mathcal{R}$ is contained in a compact set.

Proof. Let $\varphi_{N} \in \mathcal{M}$ where $N$ is a positive integer such that $\varphi_{N} \rightarrow \varphi$ as $N \rightarrow \infty$. Then

$$
\begin{aligned}
& \left|\left(\mathcal{R} \varphi_{N}\right)(n)-(\mathcal{R} \varphi)(n)\right| \\
& \leq \sum_{s=n}^{n+T}|\mathcal{G}(n, s)|\left[| A ( s ) | \left(\sum_{u=s-\tau(s)}^{s-1}|A(u)|\left|\varphi_{N}(u)-\varphi(u)\right|\right.\right. \\
& \left.\quad+\left|Q\left(s, \varphi_{N}(s-g(s))\right)-Q(s, \varphi(s-g(s)))\right|\right) \\
& \quad+|F(s)|\left|\varphi_{N}(s-\tau(s))-\varphi(s-\tau(s))\right| \\
& \left.\quad+\left|G\left(s, \varphi_{N}(s), \varphi_{N}(s-g(s))\right)-G(s, \varphi(s), \varphi(s-g(s)))\right|\right] .
\end{aligned}
$$


Since $Q, G$ are continuous, the Dominated Convergence Theorem implies,

$$
\lim _{N \rightarrow \infty}\left|\left(\mathcal{R} \varphi_{N}\right)(n)-(\mathcal{R} \varphi)(n)\right|=0,
$$

then $\mathcal{R}$ is continuous. Next, we show that the image of $\mathcal{R}$ is contained in a compact set, let $\mathcal{M}$ defined by (16), by (6) and (7), we obtain

$$
\begin{aligned}
|Q(n, y)| & \leq|Q(n, y)-Q(n, 0)+Q(n, 0)| \\
& \leq k_{1}\|y\|+|Q(n, 0)|,|G(n, x, y)| \\
& \leq|G(n, x, y)-G(n, 0,0)+G(n, 0,0)| \\
& \leq k_{2}\|x\|+k_{3}\|y\|+|G(n, 0,0)| .
\end{aligned}
$$

Let $\varphi_{N} \in \mathcal{M}$ where $N$ is a positive integer, then by (18) we obtain

$$
\begin{aligned}
\|(\mathcal{R} \varphi)(\cdot)\| & \leq c \sum_{s=0}^{T-1}\left[|A|\left(\alpha|A|+k_{1} L+\beta\right)+|F| L+\left(k_{2}+k_{3}\right) L+\gamma\right] \\
& =c T\left[|A|\left(\alpha|A| L+k_{1} L+\beta\right)+|F| L+\left(k_{2}+k_{3}\right) L+\gamma\right],
\end{aligned}
$$

where

$$
\begin{aligned}
& \alpha=\sup _{n \in[0, T-1] \cap \mathbb{Z}}|\tau(n)|, \\
& \beta=\sup _{n \in[0, T-1] \cap \mathbb{Z}}|Q(n, 0)|, \\
& \gamma=\sup _{n \in[0, T-1] \cap \mathbb{Z}}|G(n, 0,0)|, \\
& c=\sup _{n \in[0, T-1] \cap \mathbb{Z}}\left(\sup _{s \in[n, n+T-1] \cap \mathbb{Z}}|\mathcal{G}(n, s)|\right) .
\end{aligned}
$$

Second, we show that $\mathcal{R}$ maps bounded subsets into compact sets. As $\mathcal{M}$ is bounded and $\mathcal{R}$ is continuous, then $\mathcal{R} \mathcal{M}$ is a subset of $\mathbb{R}^{N T}$ which is bounded. Thus $\mathcal{R} \mathcal{M}$ is contained in a compact subset of $\mathcal{M}$. Therefore $\mathcal{R}$ is continuous in $\mathcal{M}$ and $\mathcal{R} \mathcal{M}$ is contained in a compact subset of $\mathcal{M}$.

Lemma 3.2. Suppose (4)-(6) hold and

$$
k_{1}+\alpha|A|<1 \text {. }
$$

If $\mathcal{S}$ is defined by (19), then $\mathcal{S}$ is a contraction.

Proof. Let $\mathcal{S}$ be defined by (19). Then for $\varphi_{1}, \varphi_{2} \in \mathcal{M}$ we have by (6)

$$
\begin{aligned}
\left|\left(\mathcal{S} \varphi_{1}\right)(n)-\left(\mathcal{S} \varphi_{2}\right)(n)\right|= & \mid Q\left(n, \varphi_{1}(n-g(n))\right)-Q\left(n, \varphi_{2}(n-g(n))\right) \\
& +\sum_{s=n-\tau(n)}^{n-1} A(s) \varphi_{1}(s)-\sum_{s=n-\tau(n)}^{n-1} A(s) \varphi_{2}(s) \mid \\
\leq & \left(k_{1}+\alpha|A|\right)\left\|\varphi_{1}-\varphi_{2}\right\| .
\end{aligned}
$$

Hence $\mathcal{S}$ is contraction by $(22)$. 
Theorem 3.1. Suppose the assumptions of the Lemmas 3.1 and 3.2 hold. If there exists a constant $L>0$ defined in $\mathcal{M}$ such that

$$
\begin{aligned}
& c T\left[|A|\left(\alpha|A| L+k_{1} L+\beta\right)+|F| L+\left(k_{2}+k_{3}\right) L+\gamma\right] \\
& \quad+k_{1} L+\beta+\alpha|A| L \leq L .
\end{aligned}
$$

Then (2) has a T-periodic solution.

Proof. By Lemma 3.1, $\mathcal{R}: \mathcal{M} \rightarrow \mathcal{C}_{T}$ is continuous and $\mathcal{R}(\mathcal{M})$ is contained in a compact set. Also, from Lemma 3.2, the mapping $\mathcal{S}: \mathcal{M} \rightarrow \mathcal{C}_{T}$ is a contraction. Next, we show that if $\varphi, \phi \in \mathcal{M}$, we have $\|\mathcal{R} \varphi+\mathcal{S} \phi\| \leq L$. Let $\varphi, \phi \in \mathcal{M}$ with $\|\varphi\|,\|\phi\| \leq L$. Then

$$
\begin{aligned}
\|(\mathcal{R} \varphi)(\cdot)+(\mathcal{S} \phi)(\cdot)\| \leq & c T\left[|A|\left(\alpha|A| L+k_{1} L+\beta\right)\right. \\
& \left.\quad+|F| L+\left(k_{2}+k_{3}\right) L+\gamma\right] \\
& +k_{1} L+\beta+\alpha|A| L \\
\leq & L .
\end{aligned}
$$

Clearly, all the hypotheses of the Krasnoselskii's theorem are satisfied. Thus there exists a fixed point $z \in \mathcal{M}$ such that $z=\mathcal{R} z+\mathcal{S} z$. By Lemma 2.1 this fixed point is a solution of (2). Hence (2) has a $T$-periodic solution.

Theorem 3.2. Suppose the assumptions of Lemma 2.1 hold. If

$$
c T\left[|A|\left(\alpha|A|+k_{1}\right)+|F|+\left(k_{2}+k_{3}\right)\right]+k_{1}+\alpha|A|<1,
$$

then equation (2) has a unique T-periodic solution.

Proof. Let the mapping $\mathcal{H}$ be given by (17). For $\varphi_{1}, \varphi_{2} \in \mathcal{C}_{T}$, we have

$$
\begin{aligned}
\left|\left(\mathcal{H} \varphi_{1}\right)(n)-\left(\mathcal{H} \varphi_{2}\right)(n)\right| & \mid Q\left(n, \varphi_{1}(n-g(n))\right)-Q\left(n, \varphi_{2}(n-g(n))\right) \\
\leq & +\sum_{s=n-\tau(n)}^{n-1} A(s) \varphi_{1}(s)-\sum_{s=n-\tau(n)}^{n-1} A(s) \varphi_{2}(s) \mid \\
& +\sum_{s=n}^{n+T}|\mathcal{G}(n, s)||A(s)|\left[\sum_{u=s-\tau(s)}^{s-1}|A(u)|\left|\varphi_{1}(u)-\varphi_{2}(u)\right|\right. \\
& \left.+\left|Q\left(s, \varphi_{1}(s-g(s))\right)-Q\left(s, \varphi_{2}(s-g(s))\right)\right|\right] \\
& +\sum_{s=n}^{n+T}|\mathcal{G}(n, s)|\left[|F(s)|\left|\varphi_{1}(s-\tau(s))-\varphi_{2}(s-\tau(s))\right|\right. \\
& \left.+\left|G\left(s, \varphi_{1}(s), \varphi_{1}(s-g(s))\right)-G\left(s, \varphi_{2}(s), \varphi_{2}(s-g(s))\right)\right|\right] \\
= & {\left[c T\left[|A|\left(\alpha|A|+k_{1}\right)+|F|+\left(k_{2}+k_{3}\right)\right]+k_{1}+\alpha|A|\right]\left\|\varphi_{1}-\varphi_{2}\right\| } \\
< & \left\|\varphi_{1}-\varphi_{2}\right\| .
\end{aligned}
$$

Since (23) hold, the Contraction Mapping Principle completes the proof. 
Corollary 3.1. Suppose (4) and (5) hold. Let $\mathcal{M}$ defined by (16). Suppose there are positive constants $k_{1}^{*}, k_{2}^{*}$ and $k_{3}^{*}$, such that for $x, y, z$ and $w \in \mathcal{M}$, we have

$$
\begin{gathered}
|Q(n, x)-Q(n, y)| \leq k_{1}^{*}\|x-y\| \text { and } k_{1}^{*}<1, \\
|G(n, x, y)-G(n, z, w)| \leq k_{2}^{*}\|x-z\|+k_{3}^{*}\|y-w\| .
\end{gathered}
$$

and

$$
\begin{aligned}
& c T\left[|A|\left(\alpha|A| L+k_{1}^{*} L+\beta\right)+|F| L+\left(k_{2}^{*}+k_{3}^{*}\right) L+\gamma\right] \\
& \quad+k_{1}^{*} L+\beta+\alpha|A| L \leq L .
\end{aligned}
$$

If $\|\mathcal{H} \varphi\| \leq L$, for $\varphi \in \mathcal{M}$, then (2) has a T-periodic solution in $\mathcal{M}$. Moreover, if

$$
c T\left[|A|\left(\alpha|A|+k_{1}^{*}\right)+|F|+\left(k_{2}^{*}+k_{3}^{*}\right)\right]+k_{1}^{*}+\alpha|A|<1,
$$

then (2) has a unique solution in $\mathcal{M}$.

Proof. Let the mapping $\mathcal{H}$ defined by (17). Then the proof follow immediately from Theorem 3.1 and Theorem 3.2.

Remark 3.1. Note that, when $\tau(n)=0$, the Theorems 3.1 and 3.2 reduces to the Theorems 2.5 and 2.7 respectively in [13]. The first part of the Corollary 3.1 reduces to [13, Corollary 2.6] and the second part reduces to [13, Corollary 2.8].

Example 3.1. Consider the 2-dimensional nonlinear neutral difference system

$$
\begin{aligned}
\Delta\left(\begin{array}{l}
x_{1}(n) \\
x_{2}(n)
\end{array}\right)= & \left(\begin{array}{cc}
0 & \lambda_{4} \\
-\lambda_{4} & -\lambda_{4}
\end{array}\right)\left(\begin{array}{c}
x_{1}(n-\tau(n)) \\
x_{2}(n-\tau(n))
\end{array}\right) \\
& +\Delta\left(\begin{array}{c}
0 \\
\lambda_{1} \sin (n) x_{1}^{2}(n-g(n))
\end{array}\right) \\
& +\left(\begin{array}{c}
0 \\
\lambda_{2} \cos (n) x_{1}(n)-\lambda_{3} x_{1}(n-g(n))
\end{array}\right),
\end{aligned}
$$

where

$$
\begin{gathered}
A(\cdot)=\left(\begin{array}{cc}
0 & \lambda_{4} \\
-\lambda_{4} & -\lambda_{4}
\end{array}\right), \\
Q(n, x(n-g(n)))=\left(\begin{array}{c}
0 \\
\lambda_{1} \sin (n) x_{1}^{2}(n-g(n))
\end{array}\right), \\
0 \\
G(n, x(n), x(n-g(n)))=\left(\begin{array}{c}
0 \\
\lambda_{2} \cos (n) x_{1}(n)-\lambda_{3} x_{1}(n-g(n))
\end{array}\right) .
\end{gathered}
$$

Let $\tau(n)=\alpha \in \mathbb{Z}^{+}, g(\cdot): \mathbb{Z} \rightarrow \mathbb{Z}^{+}$are nonnegative sequence and $2 \pi$ periodic. Since the matrix $B=I+A$ has eigenvalues with non-zero real 
parts, the system $x(n+1)=B x(n)$ is noncritical. So, let a Banach space $\left(\mathcal{C}_{2 \pi},\|\cdot\|\right)$,

$$
\mathcal{C}_{2 \pi}=\left\{\phi: \phi \in C\left(\mathbb{Z}, \mathbb{R}^{2}\right), \phi(n+T)=\phi(n)\right\},
$$

a closed bounded convex subset of $\mathcal{C}_{T}$,

$$
\mathcal{M}=\left\{\varphi \in \mathcal{C}_{2 \pi},\|\varphi\| \leq L\right\} .
$$

Let $\varphi=\left(\varphi_{1}, \varphi_{2}\right), \phi=\left(\phi_{1}, \phi_{2}\right)$. Then for $\varphi, \phi \in \mathcal{M}$ we have

$$
\begin{aligned}
& \|G(\cdot, \varphi(\cdot), \varphi(\cdot-g(\cdot)))-G(\cdot, \phi(\cdot), \phi(\cdot-g(\cdot)))\| \\
& \leq \lambda_{2}\|\varphi-\phi\|+\lambda_{3}\|\varphi-\phi\| .
\end{aligned}
$$

Hence $k_{2}^{*}=\lambda_{2}, k_{3}^{*}=\lambda_{3}$, in the same way $k_{1}^{*}=2 \lambda_{1} L, \beta=0, \gamma=0$ and

$$
F(n)=A(n)-(1-\Delta \tau(n)) A(n-\tau(n))=0, \quad|A|=\lambda_{4} .
$$

Consequently

$$
c T\left[\lambda_{4}\left(\alpha \lambda_{4} L+2 \lambda_{1} L^{2}\right)+\left(\lambda_{2}+\lambda_{3}\right) L\right]+2 \lambda_{1} L^{2}+\alpha \lambda_{4} L \leq L,
$$

for all $\lambda_{i}, 1 \leq i \leq 4$ small enough. Then (27) has a $2 \pi$-periodic solution, by Corollary 3.1. Moreover,

$$
c T\left[\lambda_{4}\left(\alpha \lambda_{4}+2 \lambda_{1} L\right)+\left(\lambda_{2}+\lambda_{3}\right)\right]+2 \lambda_{1} L+\alpha \lambda_{4}<1,
$$

is satisfied for $\lambda_{i}, 1 \leq i \leq 4$ small enough. Then (27) has a unique $2 \pi$ periodic solution, by Corollary 3.1 .

\section{REFERENCES}

[1] R.P. Agarwal, Difference Equations and Inequalities, Academic Press, New York, 2000.

[2] R.P. Agarwal, P.Y. Pang, On a generalized difference system, Nonlinear Anal. 30 (1997) 365-376.

[3] R.P. Agarwal, W.T. Li, P.Y.H. Pang, Asymptotic behavior of a nonlinear difference equation, J. Differ. Equ. Appl. 8 (8) (2002) 719-728.

[4] R.P. Agarwal, W.T. Li, P.Y.H. Pang, Asymptotic behavior of nonlinear difference systems, Appl. Math. Comput. 140 (2-3) (2003) 307-316.

[5] A. Ardjouni and A. Djoudi, Periodic solutions in totally nonlinear difference equations with functional delay, Stud. Univ. Babeş-Bolyai Math. 56 (2011), No. 3, 7-17.

[6] T. A. Burton, Stability by Fixed Point Theory for Functional Differential Equations, Dover Publications, New York, 2006.

[7] S. Elaydi, An Introduction to Difference Equations, Springer, New York, 1999.

[8] S. Elaydi, Periodicity and stability of linear Volterra difference systems, J. Math. Anal. Appl. 181 (1994) 483-492.

[9] P. Eloe, M. Islam, Y. Raffoul, Uniform asymptotic stability in nonlinear Volterra discrete systems, Comput. Math. Appl., Special Issue Adv. Differ. Equations IV, Volume 45, Issues 6-9, March-May 2003, Pages 1033-1039. 
[10] W.G. Kelly and A.C. Peterson, Difference Equations : An Introduction with Applications, Academic Press, 2001.

[11] V. Lakshmikantham, S.G. Deo, Methods of Variation of Parameters for Dynamical Systems, Gordon and Breach Science Publishers, Australia, 1998.

[12] M. Maroun and Y.N. Raffoul, Periodic solutions in nonlinear neutral difference equations with functional delay, J. Korean Math. Soc. 42 (2005), 255-268.

[13] Y.N. Raffoul, Existence of periodic solutions in neutral nonlinear difference system with delay, J. of Difference Eq. and Appl. Vol. 11, No. 13 (2005) 1109-1118.

[14] D.R. Smart, Fixed Point Theorems, Cambridge University Press, 1980.

\author{
Mouataz Billah Mesmouli \\ Applied Mathematics Lab \\ Faculty of Sciences \\ Department of Mathematics \\ UnIV ANNABA \\ P.O. Box 12, Annaba 23000 \\ Algeria \\ E-mail address: mesmoulimouataz@hotmail.com
}

\section{Abdelouaheb Ardjouni}

Faculty of Sciences and Technology

Department of Mathematics and Informatics

Univ Souk Ahras

P.O. Box 1553, Souk Ahras, 41000

Algeria

Address 2:

Applied Mathematics Lab

FACUlty of Sciences

Department of Mathematics

Univ AnNABA

P.O. Box 12, Annaba 23000

Algeria

E-mail address: abd_ardjouni@yahoo.fr 